\title{
Modified technique of underlay myringoplasty
}

\begin{abstract}
Objective: To determine the success rate of Myringoplasty in terms of graft uptake and hearing improvement using a modified technique.

Methods: A prospective study on 40 patients who underwent Myringoplasty by underlay technique from December 2015 to December 2017 was carried out at Tata motors Hospital, Jamshedpur. In our technique, Modified Rosen's incision was used rather than a classical canal wall incision and Tympanomeatal flap elevated all around with 360 degree elevation of annulus. Results were analyzed at 3 month. The outcomes were evaluated by canal healing, graft uptake rate, and hearing results at 3 month.

Results: A total of 40 patients, (47.5\% male and $52.5 \%$ female) between the ages 18 to 60 years of chronic otitis media inactive mucosal type with dry perforation were included in the study. The average hearing loss in pre-op patient was $38.55 \mathrm{~dB}$ which improved to $17.33 \mathrm{~dB}$ in post op patient with improvement by $21.22 \mathrm{~dB}$ at 3 month postoperatively $(\mathrm{P}<0.05)$. Graft uptake rate was $97.5 \%$. No granulations, edema noted in any of the patients postoperatively.

Conclusion: The proposed technique of Myringoplasty is a highly successful and suitable for all types of tympanic membrane perforations, also easier than classical Myringoplasty and affords an excellent graft uptake rate with satisfying hearing improvement without any specific complications.
\end{abstract}

Keywords: myringoplasty, underlay myringoplasty, modified Rosen's incision, circumferential sub annular
Volume 10 Issue 5 - 2018

\author{
Shalabh Rastogi,' Rita Pandit, ${ }^{2}$ Prasanna \\ KumarVD Vasamsetty² \\ 'Lecturer of Otorhinolaryngology Cairo University, \\ Egypt \\ ${ }^{2}$ Lecturer of Otorhinolaryngology Sohag University, Egypt
}

Correspondence: Shalabh Rastogi, Consultant and Head, Department of ENT,Tata Motors Hospital, Jamshedpur. Jharkhand, Pin 831003, India, Tel +917209000103, Email shalabh.rastogi@tatamotors.com

Received: June 19, 2018| Published: September 18, 2018

\section{Introduction}

Tympanic membrane perforation, caused by trauma, infection, post myringotomy and grommet etc., results in recurrent ear discharge and conductive hearing loss. Myringoplasty is the surgery advocated for repair of tympanic membrane perforation. Myringoplasty, is introduced by berthold, ${ }^{1}$ and was further developed by wullstein ${ }^{2}$ and zollner. $^{3}$

Two classic methods for Myringoplasty are underlay and overlay techniques each having its advantages and disadvantages. ${ }^{4-5}$ In the overlay technique, after the elevation of squamous tissue, the graft is positioned lateral to the annulus and fibrotic layer of the tympanic membrane residue. Overlay technique carries a risk of graft lateralization, anterior blunting, delayed healing, stenosis of the external canal, epithelial pearls, and iatrogenic cholesteatoma. ${ }^{6}$ Despite its higher success in repairing anterior and subtotal perforations, there is a consensus concerning the overlay technique being more technically challenging. ${ }^{7}$ The underlay technique is perhaps more commonly used worldwide, this technique is easier to perform, lower risk for lateralization, less time consuming, with more acceptable success rate even in the hands of less-experienced surgeons. In this technique, the graft is placed medial to the entire tympanic membrane remnant and also to the malleus handle and is more suitable for posterior perforations. ${ }^{8}$ This technique has disadvantages including a decreased mesotympanic space, medial displacement of the graft, synaechae formation between graft and promontory and lower success rate in subtotal and anterior perforations. ${ }^{6}$

Over the years Myringoplasty has been modified by many surgeons in term of approaches, techniques, canal wall incision, grafting. There are several ways in which canal wall incisions are placed during Myringoplasty. Each carries their own merits and demerits. We propose a modification of the traditional approach of giving canal wall incision and report high success rates, for closure of all types of perforation by underlay technique.

\section{Materials and method}

This was a prospective observational study of patients of COM undergoing Myringoplasty at Tata motors Hospital, Jamshedpur, Jharkhand, India from Dec 2015 to December 2017. Ethical clearance was taken for the study.

A total of 40 patients between the ages 18 to 60 years with central perforation of tympanic membrane which were dry for at least 3 weeks before surgery were included in the study. A detailed history was taken from each patient, along with ear examination with otoscope, examination under microscope and audiological evaluation. The exclusion criteria had been evidence of retraction pocket, or a marginal perforation, or ossicular deformity or any evidence of atticoantral disease.

\section{Surgical technique}

All patients were operated by same surgeon by post aural route using temporalis fascia graft under general anesthesia.

Post aural Wilde's incision given, temporalis fascia graft harvested meatotomy incision given $2 \mathrm{~mm}$ medial to spine of Henle to enter external auditory canal. Margins of perforation freshened. 
Modified Rosen's incision was given in canal skin as described in fig. leaving anterior skin as vascular strip which meets posteriorly to meatotomy incision. Tympanomeatal flap elevated all around along with 360 degree elevation of annulus after denuding handle of malleus. . Anteriorly annulus is lifted up from its sulcus and the anterior canal wall skin elevated using a circular knife from medial to lateral direction and Tympanomeatal skin reflected anteriorly by laterally based anterior skin flap between 2 to 4 o'clock for right ear. This way medial part of bony canal wall is visible all around. Any drilling to reduce anterior, inferior canal hump is done. Graft is placed all around over bony meatal wall medial or lateral to handle of malleus. Tympanomeatal flap is reposited back over graft maintaining anterior angle. No gel foam was placed in middle ear as graft is well supported all around. EAC packed with medicated gel foam and wound sutured (Figure 1).

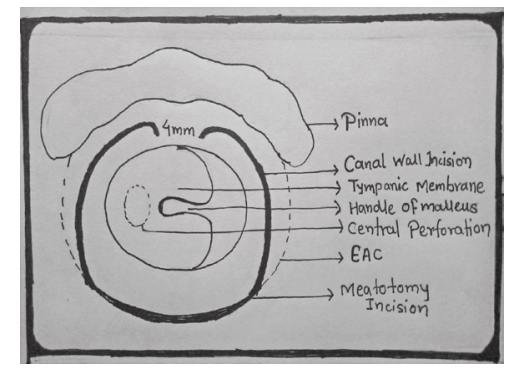

Figure I Schematic diagram of left ear showing modified Rosen's incision. Upper incision start at $4 \mathrm{~mm}$ lateral to anterior annulus at 10 o' clock curves spirally superiorly laterally and posteriorly to meet upper end of meatotomy incision. Similarly lower incision starts at $4 \mathrm{~mm}$ laterally to anterior annulus at 8 o' clock curves spirally inferiorly laterally and posteriorly to meet lower end meatotomy incision.

Patients were evaluated at 3 month post operatively for canal healing, granulations, graft uptake. Post-op audiometric evaluation was done at 3 months post-op.

\section{Statistical analysis}

All the results were analyzed from master chart considering the intact graft at 3rd month post-operative period as successful graft uptake and difference in pre-operative and post-operative average airbone gap as hearing gain. "P" value is determined using paired-t test with the help of EPI-INFO SOFTWARE 2.2.1,

http:// http://insilico.net/tools/statistics/ttest and MICROSOFT EXCEL

\section{Results}

In this study a total number of 40 cases of chronic otitis media inactive mucosal type with dry perforation were taken. Out of 40 cases, $47.5 \%$ were male and $52.5 \%$ were female. Age range was from 15 to 60 years. Average age of male patients is 30.73 years and female patients are 32.77 years. Right ear was operated in 19 (47.5\%) patients and left ear was operated in $21(52.5 \%)$ patients.

Patients were classified on the basis of degree of hearing loss. Patient were categorized in 4 groups less than $10 \mathrm{~dB}$ loss, 10 to $20 \mathrm{~dB}$ loss, 20 to $30 \mathrm{~dB}$ loss and more than $30 \mathrm{~dB}$ loss. In all the cases only conductive hearing loss was recorded in pure tone audiometry. Bone conduction up to 5 decibels is considered as test error and was not taken into consideration.
There were no granulations, edema in EAC in any of the patients at 3 months. Complete healing of canal wall was present in all the 40 cases. Graft uptake was successful in $39 / 40$ patients with success rate of $97.5 \%$ at 3 month post op period.

The avg hearing loss in preop patient is $38.55 \mathrm{~dB}$ which improves to 17.33 in post op patient with improvement by $21.22 \mathrm{~dB}$. Determining ' $\mathrm{P}$ ' value for the pre op and post op hearing comes out to be taking two sided confidence interval as 95\% (Table 1) ( Figure 2).

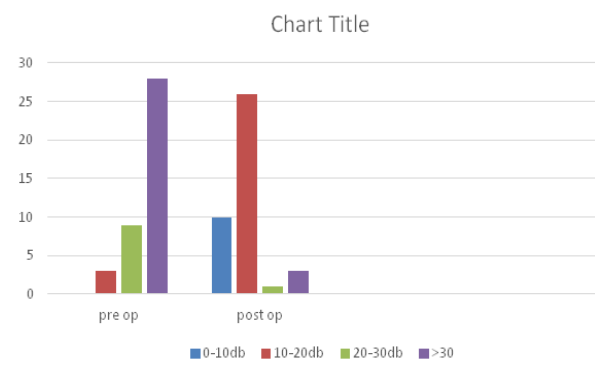

Figure 2 Showing category wise distribution of patients pre op and post op. It can be seen that in preop group more patients are there in 3rd and 4th category where as there is improvement in hearing loss in post op categories with more patients in Ist and 2nd categories.

Table I Deriving ' $p$ ' value and the significance of the study

\begin{tabular}{llll}
\hline Groups & Sample size & Mean & Standard deviation \\
\hline Pre-op & 40 & 38.55 & 10.33 \\
Post-op & 40 & 17.33 & 8.33 \\
'P' value & & $<0.05$ Significant & \\
\hline
\end{tabular}

\section{Discussion}

The ultimate goal of Myringoplasty is to re-establish a tympanic membrane. The surgical outcome of Myringoplasty is influenced by many factors which include both disease factor and surgical technique. Healing has a much poorer prognosis in cases of suspected tubal dysfunction, adhesive processes, tympanic fibrosis, bilateral disease, and defects of the entire tympanic membrane. ${ }^{9-11}$ The success of Myringoplasty also depends on the surgical technique which allow us complete eradication of the disease and recreation of a healthy, functional and aerated middle ear. ${ }^{12}$

In our study the main point of discussion are canal incision which gives surgeon good exposure of canal wall and create Tympanomeatal flap with good blood supply, 360 degree elevation of fibrous annulus from its sulcus, circumferential sub annular placement of graft on bony canal wall and no placement of gel foam in middle ear. And the success of this technique to be evaluated by healing of EAC, graft uptake and hearing improvement.

Many canal wall incisions were described in literature like Rosen's incision, Lampert 1 incision, 3 flap techniques, spiral incision by Ugo fisch. Palva ${ }^{13}$ introduced his Swing Door Technique to enhance anterior visualization of the tympanum. Ralli et al. ${ }^{14}$ used underlay anchored Myringoplasty which utilizes anterior and posterior tunnels to achieve proper tension of the tympanic membrane as well as lateral traction of the malleus handle. It resulted in drum healing (91.7\%) and pure tone average (PTA) post operatively was $27 \mathrm{~dB}$ as compared with $55 \mathrm{~dB}$ preoperatively. Primrose \& $\mathrm{Kerr}^{15}$ were able to improve graft tension by using an anterior tunnel created under the annulus. 
Gristwood \& Venables ${ }^{16}$ described an underlay Myringoplasty creating two anterior tunnels for graft stabilization. Sauvage et al., ${ }^{17}$ presented a surgical technique that included the creation of a large anterior flap for stabilizing the fascia

Most of these technique are either suitable for anterior or posterior perforation or are difficult to learn. The main aim of canal incisions is to maintain healthy blood supply to the Tympanomeatal flap, should be easy to learn and perform, can be tailor made according to canal size, should provide good view of bony canal wall to address any overhangs, should be able to give 3 to $4 \mathrm{~mm}$ of margins all around perforation to prevent rolling of margins and should be easy to handle during Tympanomeatal flap repositioning over graft. The incision we proposed as modified Rosen's incision provides all these benefits. Start point of upper incision is 3 to $4 \mathrm{~mm}$ lateral to annulus at $2 \mathrm{o}$ 'clock for right ear and spirally goes upward backward and posteriorly to meet meatotomy incision and inferior incision starts at same level from 4 o'clock for right ear goes spirally downward laterally and posteriorly to meet inferior end of meatotomy incision. There are no corners in this incision so better apposition is achieved while repositioning can easily cover wider bony canal after canal widening. The incision can be placed medially or laterally in case of small canal and wide canal respectively to provide adequate balance between bulk of Tympanomeatal flap and working space for graft placement.

The anterior annulus elevation is done by blunt circular knife to sub luxate fibrous annulus from its sulcus and sharp elevation by circular knife to cut anterior mucosa to free Tympanomeatal flap just lateral to Eustachian tube opening from medial to lateral direction. The entire TM flap is reflected laterally and anteriorly by laterally based canal skin flap between 2 to 4 o'clock for right ear. This dissection is similar to making anterior tunnel for anterior tuck technique but gives advantage of addressing anterior canal wall bulge which was not possible in anterior tunnel technique.

Annulus is raised 360 degree after dissecting flap from malleus and entire middle ear cavity is visible along with medial bony canal. Graft is then placed over canal wall. Graft can be placed medial or lateral to handle of malleus as per ease. Tympanomeatal flap is repositioned over graft. Anterior angle should be maintained with help of dry gel foam placed lateral to Tympanomeatal flap. Since graft is sandwiched between canal wall and Tympanomeatal flap it does not require any gel foam support from medial side and hence no gel foam is to be placed in middle ear. This gives additional advantage of early hearing gain and prevents any fibrosis in middle ear. Iraninan author Samad et al., ${ }^{18}$ also advocated no gel foam to be used in middle ear ${ }^{18}$ for advantage of early hearing improvement and preventing the risk of middle ear fibrosis.

In our study no granulations or canal wall sagging were found on pack removal on $10^{\text {th }}$ post op day and canal wall was nicely healed by that time. Canal edema settles down by 2 to 3 weeks post operatively. Results obtained were similar to the study done by Roychaudhury ${ }^{19}$ and Vaidya. ${ }^{20}$

The success rate of graft uptake of $97.5 \%$ percent in our study which is similar to many other techniques of Myringoplasty and considered as good uptake rate. The only case in which graft uptake was not successful was a result of otitis media in early post op period resulting in re perforation. Study done by Murugendrappa MA et al., ${ }^{21}$ had shown $96 \%$ success rate. Mokhtarinejad et al.,22 reported $95 \%$ success in graft uptake by circumferential sub annular graft technique although his mean air-bone gap ( $\mathrm{ABG}$ ) had reduced postoperatively. There were no cases of anterior blunting and graft lateralization in both groups. Raghuwanshi et al., ${ }^{23}$ the graft take rate was $93.75 \%$ without any retraction pockets or displaced grafts for Single stage bilateral type1 tympanoplasty. Khandelwal et al., ${ }^{24} 94.5 \%$. Schraff et al. ${ }^{25}$ described the modified window shade technique in that the anterior fibrous annulus was mobilized of its bony sulcus their overall success rate was $94.5 \%$. There were no cases of tympanic membrane lateralization or significant blunting.

In our study we got hearing improvement with A-B gap closure of less than $20 \mathrm{~dB}$ in almost 90 percent patients. Hearing gain is statistically significant in all types of tympanic membrane perforations including smaller, larger and subtotal perforations with ' $\mathrm{P}$ ' value less than 0.05 . Hearing gain analyzed as difference between preoperative and post-operative air-bone gap came as 21.22 decibel. The 'p'value came significant in respect to hearing gain with the surgical intervention. Results were similar to study by Schraff et al which showed $82 \%$ of patients demonstrated closure of their ABG to within $10 \mathrm{~dB}, 16 \%$ closed the gap to within $20 \mathrm{~dB}$, and $2 \%$ closed the gap to within $30 \mathrm{~dB} .{ }^{25}$

\section{Conclusion}

The proposed technique can be used for all type of perforation, gives good results of graft uptake and hearing improvement, does not require special skills, easy to learn and perform even for novice ear surgeon. No specific complications were encountered while using this modification of the traditional approac

\section{Compliance with ethical standards}

1. Disclosure of potential conflicts of interest-none

2. Research involving Human Participants and/or Animals-This article does not contain any studies with human participants or animals performed by any of the authors."

\section{Conflict of interest}

Authors Dr. Shalabh Rastogi, Dr. Rita Pandit, Dr. Prasanna Kumar V D Vasamsetty declares that we had no conflict of interest.

\section{References}

1. Berthold E. Ueber myringoplastik. Wier Med Bull. 1878;1:627-627.

2. Wullstein H. Theory and practice of myringoplasty. Laryngoscope. 1956;66(8):1076-1093.

3. Zollner F. The principles of plastic surgery of the sound-conducting apparatus. J Laryngol Otol. 1955;69(10):637-652.

4. Rizer FM. Overlay versus underlay tympanoplasty. Part I: historical review of the literature. Laryngoscope. 1997;107(12 Pt 21):1-25.

5. Wehrs RE. Grafting techniques. Otolaryngol Clin $N$ Am. 1999;32(3):443-455

6. Kulduk E, Dundar R, Soy FK, et al. Treatment of Large Tympanic Membrane Perforations: Medial to Malleus Versus Lateral to Malleus. Indian Journal of Otolaryngology and Head \& Neck Surgery. 2015;67(2):173-179.

7. Gersdorff M, Gerard J, Thill M. Overlay versus underlay tympanoplasty: comparative study of 122 cases. Rev Laryngol Otol Rhinol. 2003;124(1):15-22.

8. Lee HY, Auo HJ, Kang JM. Loop overlay tympanoplasty for anterior or 
subtotal perforations. Auris Nasus Larynx. 2010;37(2):162-166.

9. Onal K, Uguz MZ, Kazikdas KC, et al. A multivariate analysis of otological, surgical and patientrelated factors in determining success in myringoplasty. Clinical Otolaryngology. 2005;30(2):115-120.

10. Uguz MZ, Onal K, Kazikdas KC, et al. The influence of smoking on success of tympanoplasty measured by serum cotinine analysis. European Archives of Oto-Rhino-Laryngology. 2008;265(5):513-516.

11. Mürbe D, Zahnert T, Bornitz M, et al. Acoustic properties of different cartilage reconstruction techniques of the tympanic membrane. Laryngoscope. 2002;112(10):1769-1776.

12. Nankivell PC, Pothier DD. Surgery for tympanic membrane retraction pockets. Cochrane Database of Systematic Reviews. 2010;7.

13. Palva T. Middle ear surgery in Northern Europe. Arch Otolaryngol. 1963;78:363-370.

14. Ralli. Anchored Myringoplasty: The laryngoscope. 2000;110:674-679.

15. Primose WJ, Kerr AG. The anterior perforation. Clin Otolaryngol. 1986;11:175-176.

16. Gristwood R, Venables W. Factor influencing the outcome in type 1 tympanoplasty. Aust J Otolaryngol. 1993;4:319-331.

17. Sauvage JP, Heutebise F, Pugraud SH. Myringoplasty (technique, results). Rev Larayngol Otol Rhinol. 1998;117(3):247-251.

18. Samad G, Tootonochi SJ. Tympanoplasty without use of gel-foam in the middle ear. Iranian Journal of Otorhinolaryngology. 2008;20(52):6570

19. Roychaudhuri BK. 3-flap tympanoplasty-A simple and sure success technique. Indian J Otolaryngol Head Neck Surg. 2004;56(3):196-200.

20. Vaidya S, Sharma JK, Singh G. Study of Outcome of Tympanoplasties in Relation to Size and Site of Tympanic Membrane Perforation. Indian Journal of Otolaryngology and Head \& Neck Surgery. 2014;66(3):341346.

21. Murugendrappa MA, Siddappa PN, Shambulingegowda A, et al. Comparative Study of Two Different Myringoplasty Techniques in

Mucosal Type of Chronic Otitis Media. J Clin Diagn Res. 2016;10(2): MC01-MC03.

22. Mokhtarinejad F, Okhovat SA, Barzegar F. Surgical and hearing results of the circumferential subannular grafting technique in tympanoplasty: A randomized clinical study. Am J Otolaryngol. 2012;33:75-79.

23. Raghuwanshi SK, Asati DP. Outcome of single-Sitting Bilateral Type 1 Tympanoplasty in Indian Patients. Indian J Otolaryngo Head Neck Surg. 2013;65(Suppl 3):622-626.

24. Khandelwal G. Tympanoplasty: blood soaked gelfoam verses antibiotic-soaked gelfoam as ear canal packing. J Evolution Med Dent Sci. 2016;5(53):3560-3562.

25. Schraff S, Dash N, Strasnick B. Window shade tympanoplasty for anterior marginal perforations. Laryngoscope. 2005;115:1655-1659. 